\title{
Utilization of Transfer Learning Model in Detecting COVID-19 Cases From Chest X-Ray Images
}

\author{
Malathy Jawahar, Central Leather Research Institute, Chennai, India \\ (iD) https://orcid.org/0000-0001-6865-2097 \\ L. Jani Anbarasi, Vellore Institute of Technology, Chennai, India \\ Prassanna Jayachandran, Vellore Institute of Technology, Chennai, India \\ Manikandan Ramachandran, SASTRA University, Thanjavur, India \\ (iD https://orcid.org/0000-0001-6116-2132
}

Fadi Al-Turjman, Near East University, Nicosia, Turkey

\begin{abstract}
Diagnosis of COVID-19 pneumonia using patients' chest $\mathrm{x}$-ray images is new but yet important task in the field of medicine. Researchers from different parts of the globe have developed many deep learning models to classify COVID-19. The performance of feature extraction and classifier plays a vital role in the recognizing the different patterns in the image. The pivotal process is the extraction of optimum features from the chest x-ray images. The main goal of this study is to design an efficient hybrid algorithm that integrates the robustness of MobileNet (using transfer learning approach) to extract features and support vector machine (SVM) to classify COVID-19. Experiments were conducted to test the proposed algorithm, and it was found to have a high classification accuracy of $95 \%$.
\end{abstract}

\section{KEYWORDS}

Accuracy, COVID-19, Deep Learning Network, MobileNet, SVM

\section{INTRODUCTION}

Corona Virus Disease 2019 (COVID-19) is a new virus that was first discovered to be affecting humans in 2019 and it was found to be related to the same family of viruses as Severe Acute Respiratory Syndrome (SARS) (Lancet, 2020). It is a contiguous virus that have made its first appearance in Wuhan, China. Currently (as of 28 November 2020) 14,524,141 have died worldwide due to this virus. The rapid spread of this virus worldwide has resulted in a pandemic. India registered the first COVID-19 case on January 30 in Kerala and by February 03 two more cases were reported from the same city. In all three cases the infected individuals were students who have just returned back to India from Wuhan, China. The virus makes the immune system weaker which can eventually lead to death (Lancet, 2020; Razai et al., 2020). The virus is illustrated in Figure 1 and as it can be seen, the virus contain spikes on the crown of its outer surface which helps it in establishing a secured connection with human's airway cells (Texas, n.d.). As of November 28, 2020; 62,165,882 people were infected worldwide and 42,951,570 have recovered (Jaiswal et al., 2019; Peng et al., 2020) in the world. 


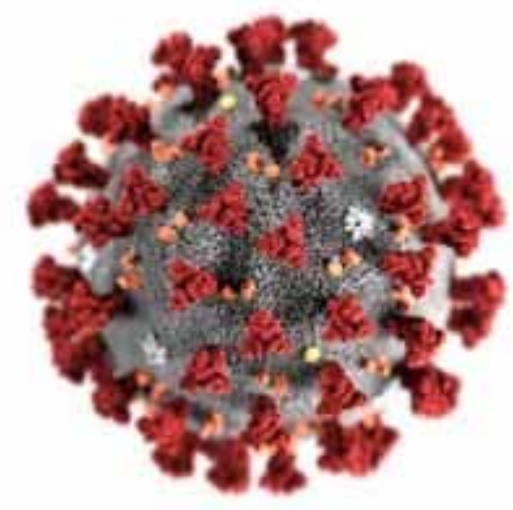

COVID-19 can spread often by physical touch between two individuals. In general, it is possible for an infected induvial to infect other healthy individuals through breath, hand contact, touch, or mucosal contact (Peng et al., 2020). As the virus make its way to the lungs, infected individuals can suffer from pneumonia. Due to the extreme shortage of the expensive test kit, Real-time polymerase chain reaction (RT-PCR) (Xie et al., 2020) has further aggravated the situation. Hence, people with possible signs of pneumonia were prescribed a chest scan such as Computer Tomography (CT) scans and X-Rays to quickly diagnose and detect whether they are infected with COVID-19 or not. The presence of COVID-19 can be automatically detected with CT scans combined with deep learning techniques (Gozes et al., 2020; Li et al., 2020). In the recent years deep learning have demonstrated a promising result in various fields of research. For the medical field in particular, deep learning methodologies were found to deliver an improved accuracy on detecting different diseases using a data set of images such as images of chest X-Ray, retina image, and brain MRI (Mahmud et al., 2020; Mahmud et al., 2018). X-Ray machines are very useful as they offer a feasible and faster means of detecting disease through the process of scanning different human organs.

A professional radiologist usually performs the interpretation of different X-Ray images manually. These acquired images can be trained using deep learning models to assist medical experts in detecting patients with COVID-19. The detection of individuals with COVID-19 using deep learning is especially important when the X-Ray facility is available but there is a shortage in professional radiologist in the hospital. With that being said, developing a deep learning model to detect COVID-19 cases is a challenging task due to the scarcity of COVID-19 X-Ray images (Chakraborty et al., 2015; Garg et al., 2020; Muhammad et al., 2021). Thus, it is extremely difficult to efficiently train a very deep network. On the other, transfer-learning models are found to provide a feasible solution and they are widely implemented in several recently proposed solutions (Sethy \& Behera, 2020). The conventional transfer-learning method, which uses deep networks pre-trained on ImageNet database to transfer its preliminary learning, cannot be the best option because the characteristics of COVID-19 patient's chest $\mathrm{X}$-Ray images may vary from other applications. Consequently, feature engineering and classification plays a vital role in image processing applications, especially in this case. Conventional hand-crafted feature extraction and selection is time consuming process. Deep learning architectures have been proven to be efficient in feature extraction for many applications (Sethy \& Behera, 2020). The major contribution of this research work is to investigate the implementation of feature engineering method through knowledge transfer approach of combining the deep learning MobileNet model trained in different domain to a domain specific such as COVID-19 data set. SVM classifier was trained and tuned 
with the extracted features to accelerate the classification process. Additionally, this research work will compare the accuracy of the traditional deep learning models with the proposed hybrid model. This research paper is structured as follows: Section 2 discusses the existing work in the automatic classification of COVID-19. Section 3 explains the proposed model and the developed architecture. Section 4 provides results and discussion on the conducted experiments. Section 5 summarizes the key findings of this work along with recommendations on a related future scope of work.

\section{LITERATURE REVIEW}

Machine learning algorithms (Ibrahim et al., 2020; Rasheed, 2020) extracts features to efficiently exploit features of interest from chest X-ray images to efficiently diagnose pneumonia. Features like mean, mode and kurtosis, are extracted from the chest X-rays and are classified using Multi-Layer Perceptron (MLP), logistic regression and random forests. Ambita et al. (Ambita et al., 2020) analyzed Chest X-rays (Al-Turjman \& Deebak, 2020; Kolhar et al., 2020; Srivastava, 2020) using Support Vector Machine (SVM) and adaptive regression kernel descriptors for pneumonia detection. Khatri et al. (Khatri et al., 2020) identified Earth Mover's Distance (EMD) for differentiating pneumonia and non-pneumonia from chest X-rays. Santos et al. (Varela-Santos \& Melin, 2020) have suggested a methodology that determines Gray Level's texture-based statistical features Co-occurrence Matrix (GLCM) and classified using neural network. Several deep learning experiments used chest X-ray image data to identify the disease (Jaiswal et al., 2019). The pneumonia X-ray images were classified using deep learning models (Baltruschat et al., 2019), namely fine-tuned, without fine-tuning, and the scratch-trained model. This model categorized the dataset into labels like age, gender, etc., by using the ResNet model. They also used the MLP as a method of classification and obtained 82.2\% accuracy on average. The Competitive Network and Backpropagation Neural Network were used by Rahib Abyayev et al. (Abiyev \& Ma'aitah, 2018) to identify pneumonia.

The most prominent area of research for COVID-19 identification is based on transfer learning. Such works assess the levels of model fitting through customizing standard architectures, for COVID-19 detection. Apostolopoulos et al. (Apostolopoulos et al., 2020) used a transfer learning approach MobileNetV2 for classifying X-rays into multi class classifications. Farooq and Hafeez analyzed ResNet50 for classifying COVID-19 by modifying the training parameters (Farooq \& Hafeez, 2020). Apostolopoulos and Mpesiana (Apostolopoulos \& Mpesiana, 2020) performed InceptionNet, VGG19, XceptionNet, MobileNetV2 and Inception ResNetV2 for the chest X-ray classification by fine tuning parameters. Khan et al. (Khan et al., 2020) used XceptionNet for classifying COVID-19 from Chest X-rays by fine tuning the dropout layer. Mangal et al. designed CheXNet Convolutional Neural Network (CNN) (Mangal et al., 2020) by utilizing DenseNet121 as the transfer learning model. Using a deep learning model, Ioannis et al. (Apostolopoulos \& Mpesiana, 2020) and Ying et al. (Song et al., 2020) obtained an accuracy of $98.75 \%$ and $86 \%$, respectively. On the other hand, 3D CNN was used by Zheng et al. (Zheng et al., 2020) and the accuracy was found to be $90.8 \%$.

\section{PROPOSED MODEL}

It is evident from the literature review that various research works has been carried out recently for the sake of detecting the COVID-19 cases accurately using chest X-ray images. Several studies have developed models using machine learning, deep learning, and transfer learning based on computer vision algorithms. In this research work, MobileNet and SVM are used to develop a hybrid model that aims at detecting COVID-19 cases accurately and efficiently using a chest X-ray image.

\subsection{MobileNet}

MobileNet is designed mainly from depth wise convolutions filters which are later used in inceptions to reduce computation time. This depth wise filter is a form of factorized convolutions that factorizes 
the convolutions into depth wise and $1 \mathrm{x} 1$ point convolution. Each layer applies a single filter. The depth wise results are generated by the pointwise convolution. This includes two layers one for filtering and next one is for combining which result in reduction in the size and computation time. Figure 2 shows how a standard convolution is converted into depth wise and pointwise convolution.
(a) Standard Convolution Filters
(b) Depth wise Convolution Filters
(c) 1 X 1 Convolution Filters - Pointwise Convolution

As it can be seen from Figure 2, the main advantage of MobileNet is the application of a single filter to each input channel as indicated in the depth wise convolution channels. The pointwise convolution applies $1 \times 1$ convolution filter to merge the output of all the depth wise convolution.

Figure 2. shows the flow of MobileNet architecture

(a) Standard Convolution Filters
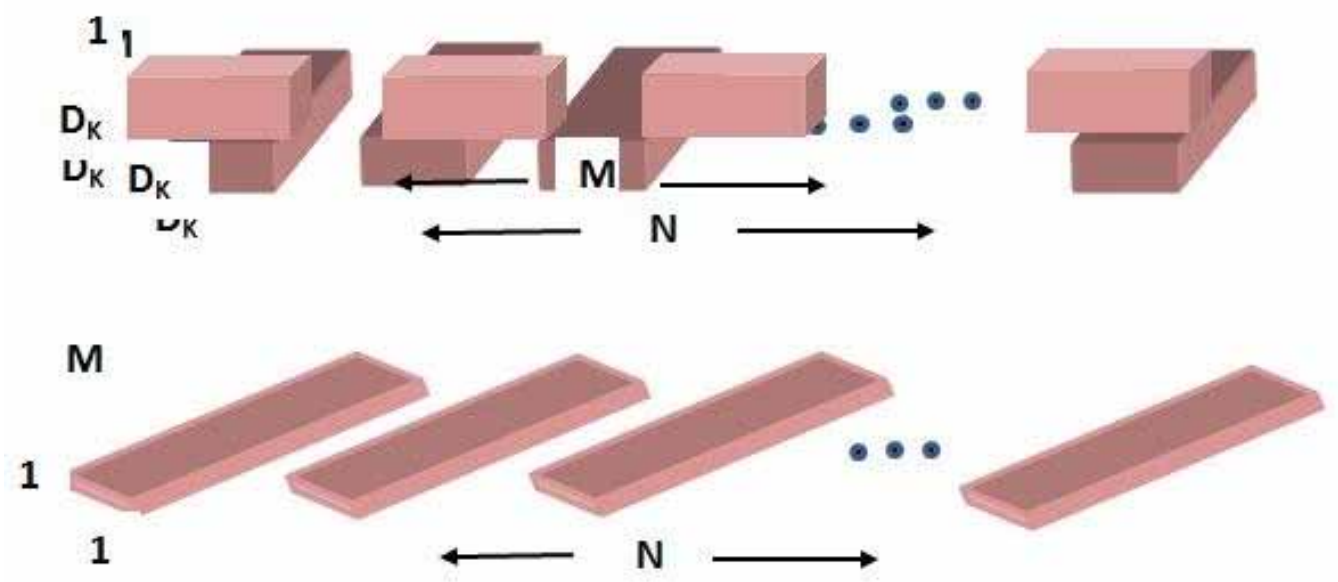

(b) Depth wise Convolution Filters

(c) 1 X 1 Convolution Filters - Pointwise Convolution

The input of the standard convolution is defined as $\mathrm{C}_{\mathrm{F}} \mathrm{XC}_{\mathrm{F}} \mathrm{XN}$ where $\mathrm{C}_{\mathrm{F}}$ is the convolution feature map with $\mathrm{F}$ feature maps and $\mathrm{N}$ inputs resulting in $\mathrm{C}_{\mathrm{o}} \mathrm{XC}_{\mathrm{o}} \mathrm{XM}$ where $\mathrm{C}_{\mathrm{o}}$ refers output feature map with 'o' feature maps with $\mathrm{M}$ outputs. The output feature map of standard convolution is given by equation 1, 2 and 3. ' $k$ ', 'l', 'n' refers to kernel size, number of output channels

$$
\mathrm{C}_{\mathrm{k}, 1, \mathrm{n}}=\sum_{\mathrm{a}, \mathrm{b}, \mathrm{z}} \mathrm{O}_{\mathrm{a}, \mathrm{b}, \mathrm{z}, \mathrm{n}} \cdot \mathrm{F}_{\mathrm{k}+1-1,1, \mathrm{j}-1, \mathrm{z}}
$$

Depth wise convolution along with filtering is given as 
$\hat{C}_{\mathrm{k}, \mathrm{l}, \mathrm{n}}=\sum_{\mathrm{a}, \mathrm{b}, \mathrm{z}} \hat{O}_{\mathrm{a}, \mathrm{b}, \mathrm{z}, \mathrm{n}} \cdot \mathrm{F}_{\mathrm{k}+1-1,1, \mathrm{j}-1, \mathrm{z}}$

Where $\hat{O}$ refers the depthwise kernel layer. MobileNet uses 3 X 3 depth wise separable convolutions achieving 9 to 10 times less computation time. The reduction of computation is achieved by the equation 3 by expressing the convolution as a two step process.

$$
\frac{\mathrm{D}_{\mathrm{O}} \mathrm{XD}_{\mathrm{F}} \mathrm{XZXD}_{\mathrm{O}} \mathrm{XD}_{\mathrm{F}}+\mathrm{ZXNXD}_{\mathrm{F}} X \mathrm{D}_{\mathrm{F}}}{\mathrm{D}_{\mathrm{O}} \mathrm{XD}_{\mathrm{O}} \mathrm{XZXNXD}_{\mathrm{F}} \mathrm{XD}_{\mathrm{F}}}
$$

The advantage of MobileNet architecture over CNN architecture is represented in the smaller computational network which results in less computation (Krause et al., 2015; Krizhevsky et al., 2012).

\subsection{Support Vector Machine}

Initially, SVMs were proposed for classifying the data and later prolonged for regression and learning process. Binary classifier using SVM resulted in either positive or negative results. It was later improved by merging the multi-class binary classifier. In addition, the SVM can also be used to map the input space into nonlinear cases. The linear classification is made equivalent to a nonlinear classification with the input space. The input feature can be mapped to a higher feature dimensions through maximal hyperplanes in SVM. Based on the kernel parameters and hyper parameters better accuracy can be achieved. The kernel parameters plays an important role in optimizing the data and identifying the best model (Szegedy et al., 2016).

\section{RESULTS AND DISCUSSION}

This section includes a complete overview of the dataset used during the experimentation process, feature extraction, model training, and validation. The performance of this model is evaluated and compared with other existing models and it is presented in the following sub-section. Figure 3 shows the proposed flow diagram this model utilizes to classify COVID-19 chest X-ray images.

\subsection{Data Acquisition and Pre-Processing}

The dataset used for the proposed work includes COVID-19 radiography images acquired from Kaggle (Kaggle, n.d.). The dataset contains a total of 535 images Where 219 of the images in the dataset are for individuals who have tested positive for COVID-19 and the remaining 316 of the images represents healthy individuals. Figure 4 shows a sample of COVID-19 images in which the arrows highlights the infected parts of the lungs.

\subsection{Model Training and Validation}

The proposed model is trained with the augmented chest X-ray images to analyze the pre-trained models. A prerequisite for the deep learning models is a large and labelled dataset to be used for training. The main constrain for the medical dataset is the availability of dataset and the expert classification to label the dataset. The open dataset available in Kaggle has a sufficient amount of Non-COVID-19 labelled images but a very small amount of COVID-19 labelled images. Hence, to avoid the unbalanced dataset issue, all 219 COVID-19 images along with a random selection of 316 Non-COVID-19 images are taken for training. A knowledge transfer learning method using MobileNet is experimented with various settings and finally the parameters are fine-tuned with the values as given in Table 1. The implementation of the MobileNet model was carried out using Keras library. 


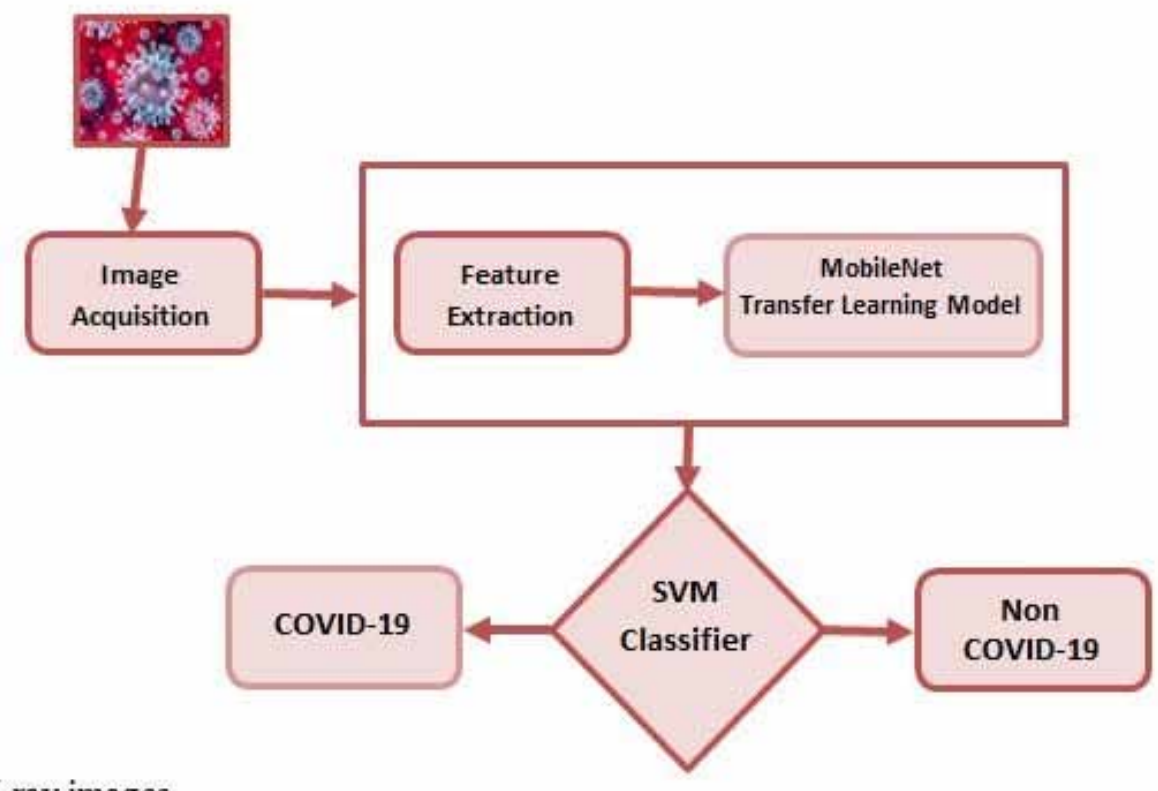

chest X-ray images.

Table 1. Experimental setting for MobileNet Variable Parameters

\begin{tabular}{|c|c|}
\hline Variables & Fine-Tuned values \\
\hline Input Size & $224 \times 224 \times 3$ \\
\hline Batch Size & 32 \\
\hline Learning Rate & 0.001 \\
\hline Learning rate decay & 0.5 \\
\hline Training Epochs & 50 \\
\hline Optimizer & ADAM \\
\hline
\end{tabular}

The model was trained for 50 epochs using ADAM optimizer with learning rate of 0.001 . Figure 5 illustrates the learning process recorded by the proposed model with respect to the number of epochs, the training and validation curves of the model accuracy and loss are represent in (a) and (b), respectively. It is evident from Figure 5 that the training and validation accuracy stabilizes well after 40 epochs. The convergence of the training and validation accuracy shows that the model has a good generalization ability to extract features corresponding to COVID-19 and Non-COVID-19 images.

The features are generated using the MobileNet transfer learning model and the features are classified using SVM classifier. 
Figure 4. Chest CT scan for individuals infected with COVID-19 (Ambita et al., 2020)

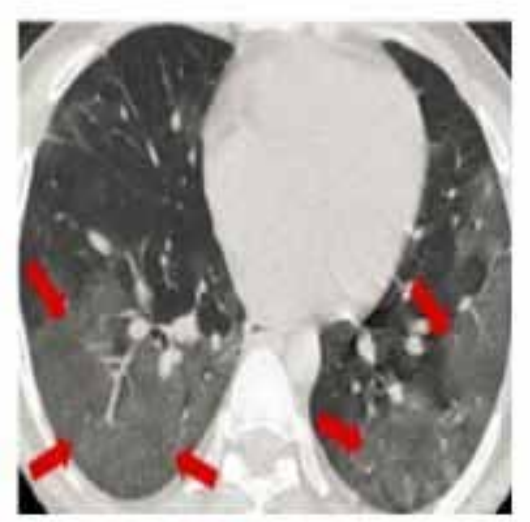

A

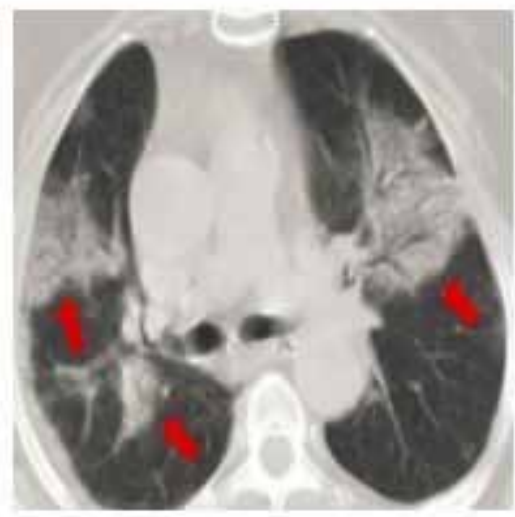

$\mathrm{C}$

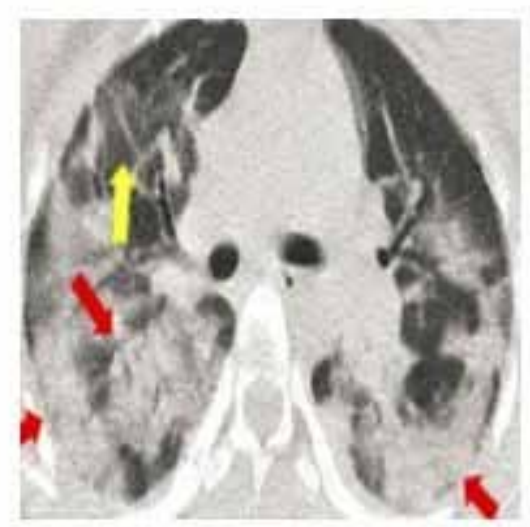

B

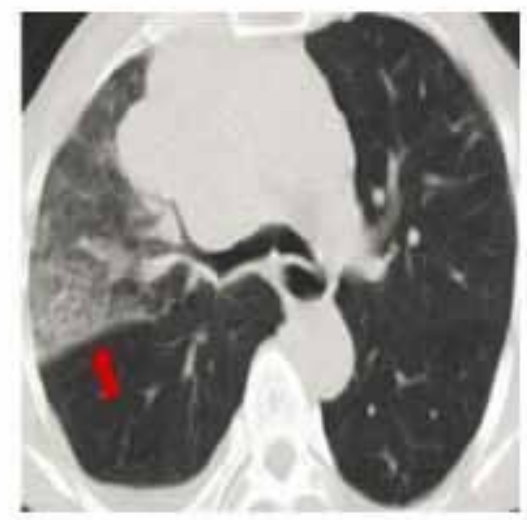

D

Figure 5. Learning process recorded by the proposed model with respect to the number of epochs.

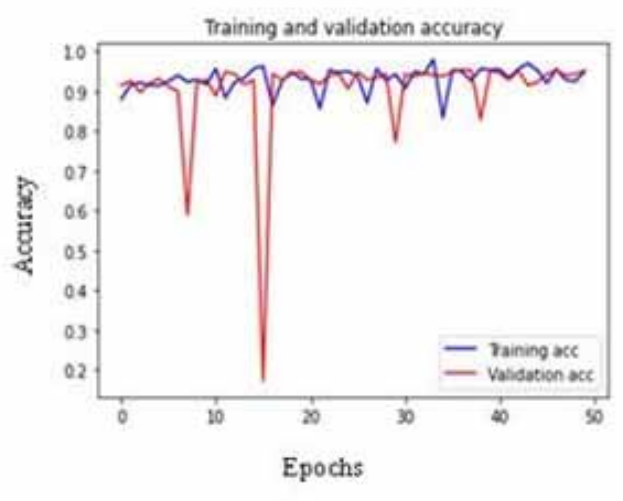

(a)

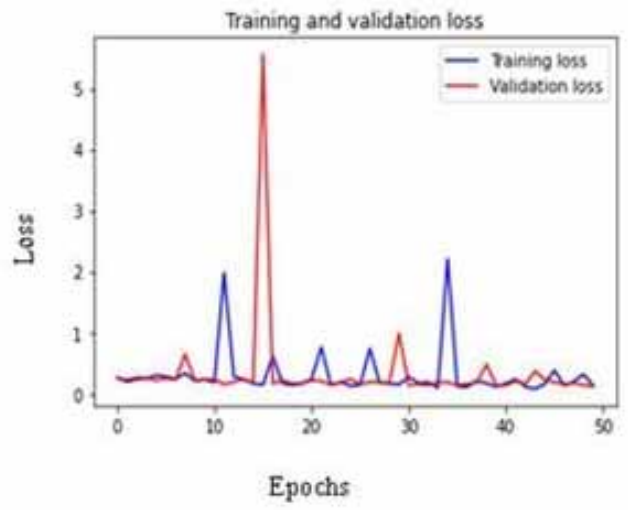

(b) 


\subsection{Effectiveness of the Proposed Architecture}

The effectiveness of the proposed architecture is analyzed using the accuracy, sensitivity, and the specificity performance evaluation metrics.

\subsubsection{Accuracy}

Accuracy is the frequency of which the predicted value matches with the actual value divided by total prediction made. The expression for accuracy is given by equation (4). TN refers to True Negative and TP refers to True Positive. FN and FP refers the False Negative and False Positive which are the model incorrect predictions.

$$
\text { Accuracy }=\frac{\mathrm{TN}+\mathrm{TP}}{\mathrm{TN}+\mathrm{FN}+\mathrm{TP}+\mathrm{FP}}
$$

\subsubsection{Sensitivity and Specificity}

The percentage of correctly identified positive data is called sensitivity whereas the percentage of correctly identified negative data is called specificity. In this case, the percent of images that are correctly identified as COVID-19 positive is called sensitivity and the percent of images that are correctly identified as Non-COVID-19 is called specificity and they are represented by equation 5 and 6 , respectively.

Sensitivity $=\frac{\mathrm{TP}}{\mathrm{TP}+\mathrm{FN}}$

$$
\text { Specificity }=\frac{\mathrm{TN}}{\mathrm{TN}+\mathrm{FP}}
$$

\subsubsection{Results Comparison}

Table 2 illustrates the comparison between the proposed hybrid model and the alternative CNN models suggested by other researchers. It is evident from Table 2 that the proposed hybrid model of MobileNet+SVM algorithm have achieved the highest accuracy (95\%), sensitivity (91\%) and specificity (97\%). For a class imbalanced medical dataset, it is critical to analyze the performance of the model in terms of Sensitivity and Specificity apart from the accuracy. It is observed that

Table 2. Comparison of the proposed hybrid model with other CNN models

\begin{tabular}{|c|c|c|c|c|}
\hline Ref & Model & Accuracy & Sensitivity & Specificity \\
\hline$[27]$ & Inception & 86.13 & 12.94 & 99.70 \\
\hline$[28]$ & Xception & 85.57 & 75.00 & 89.00 \\
\hline$[27]$ & Inception ResNet v2 & 84.38 & 55.00 & 96.00 \\
\hline $\begin{array}{c}\text { Proposed } \\
\text { Hybrid Model }\end{array}$ & MobileNet + SVM & 95 & 91.00 & 97.00 \\
\hline
\end{tabular}


the Inception and Inception with ResNetV2 shows the high specificity but a poor Sensitivity. This shows that the model does not have a good generalization ability to classify both COVID-19 and Non-COVID-19 cases. Xception network model ranks second in terms of accuracy, specificity, and sensitivity measures. The proposed hybrid algorithm fusing the efficient MobileNet and SVM classifier have outperformed the other models in terms of high classification accuracy as well as both sensitivity and specificity metrics.

Additionally, the MobileNet model has only 4.2 million parameters and 569 million multi-adds when compared to the 23.2 million parameters and 5000 multi-adds for Inception as shown in Table 3. This shows the significant computational efficiency of the MobileNet model when compared to other CNN models.

Table 3. MobileNet comparison with Inception

\begin{tabular}{|c|c|c|}
\hline Model & $\begin{array}{c}\text { Million } \\
\text { Multi- Adds }\end{array}$ & Million parameters \\
\hline Inception & 5000 & 23.2 \\
\hline MobileNet & 569 & 4.2 \\
\hline
\end{tabular}

Hence, the performance of the proposed hybrid algorithm confirms the promising potential to diagnose SARS-Cov-2 precisely.

\section{CONCLUSION}

In this paper, a hybrid algorithm that integrates MobileNet and SVM classifier was proposed in detecting COVID-19 cases using chest X-ray image. The dataset used in training the model included 219 COVID-19 cases and 316 non-COVID-19 cases. The issue of unbalanced dataset size was addressed by utilizing knowledge transfer learning using MobileNet and it was carried out using Keras library. It was evident from the training and validation accuracy curves that stabilizes well after 40 epochs indicating a good generalization ability to extract features corresponding to COVID-19 case images. The extracted features were then classified using SVM. The performance and effectiveness of the proposed algorithm have been evaluated using three different metrics including accuracy, sensitivity, and specificity. The evaluation results were then compared to other models including Inception, Inception ResNet v2 and Xception from literature sources. From the comparison it was evident that the proposed model has outperformed the rest of the compared models. The proposed model resulted in accuracy of $95 \%$, sensitivity of $91 \%$ and specificity of $97 \%$. Although the Inception model had a higher accuracy of $99.7 \%$ however the sensitivity was only $12.94 \%$ making difficult to generalize its ability in classifying the COVID-19 cases accurately. Additionally, the proposed MobileNet model was found to have only 4.2 million parameters and 569 million multi-adds compared to 23.2 million parameters and 5000 multi-adds for the Inception model. Hence, a significant computational efficiency was observed in the proposed MobileNet model compared to other CNN models. Overall, the proposed hybrid model delivered a promising result in detecting COVID-19 cases which can be potentially used in hospitals where a professional radiologist may be scarce but $\mathrm{X}$-ray is available. Directions for future work include exploring deep learning architecture namely one shot/fewer shot for smaller dataset and to tackle the imbalanced dataset challenge. 


\section{REFERENCES}

Abiyev, R. H., \& Ma'aitah, M. K. S. (2018). Deep convolutional neural networks for chest diseases detection. Journal of Healthcare Engineering, 2018, 2018. doi:10.1155/2018/4168538 PMID:30154989

Al-Turjman, F., \& Deebak, D. (2020). Privacy-Aware Energy-Efficient Framework using Internet of Medical Things for COVID-19. IEEE Internet of Things Magazine., doi:10.1109/IOTM.0001.2000123

Ambita, A. A. E., Boquio, E. N. V., \& Naval, P. C. (2020, March). Locally Adaptive Regression Kernels and Support Vector Machines for the Detection of Pneumonia in Chest X-Ray Images. In Asian Conference on Intelligent Information and Database Systems (pp. 129-140). Springer. doi:10.1007/978-3-030-42058-1_11

Apostolopoulos, I. D., Aznaouridis, S. I., \& Tzani, M. A. (2020). Extracting possibly representative COVID-19 Biomarkers from X-Ray images with Deep Learning approach and image data related to Pulmonary Diseases. Journal of Medical and Biological Engineering, 1. PMID:32412551

Apostolopoulos, I. D., \& Mpesiana, T. A. (2020). Covid-19: automatic detection from x-ray images utilizing transfer learning with convolutional neural networks. Physical and Engineering Sciences in Medicine, 1.

Baltruschat, I. M., Nickisch, H., Grass, M., Knopp, T., \& Saalbach, A. (2019). Comparison of deep learning approaches for multi-label chest X-ray classification. Scientific Reports, 9(1), 1-10. doi:10.1038/s41598-01942294-8 PMID:31011155

Chakraborty, C., Gupta, B., \& Ghosh, S. K. (2015). Identification of Chronic Wound Status under Tele-Wound Network through Smartphone. International Journal of Rough Sets and Data Analysis, 2(2), 58-77. doi:10.4018/ IJRSDA.2015070104

Chollet, F. (2017). Xception: Deep learning with depthwise separable convolutions. In Proceedings of the IEEE conference on computer vision and pattern recognition (pp. 1251-1258). doi:10.1109/CVPR.2017.195

Farooq, M., \& Hafeez, A. (2020). Covid-resnet: A deep learning framework for screening of covid19 from radiographs. arXiv preprint arXiv: 2003.14395.

Garg, L., Chukwu, E., Nasser, N., Chakraborty, C., \& Garg, G. (2020). Anonymity preserving IoT-based COVID-19 and other infectious disease contact tracing model. IEEE Access: Practical Innovations, Open Solutions, 8, 159402-159414. doi:10.1109/ACCESS.2020.3020513

Gozes, O., Frid-Adar, M., Greenspan, H., Browning, P. D., Zhang, H., Ji, W., . . Siegel, E. (2020). Rapid ai development cycle for the coronavirus (covid-19) pandemic: Initial results for automated detection \& patient monitoring using deep learning ct image analysis. arXiv preprint arXiv:2003.05037.

Ibrahim, A., Ozsoz, M., Serte, S., Al-Turjman, F., \& Yakoi, P. S. (2020). Pneumonia Classification using Deep Learning from Chest X-ray Images during COVID-19. Cognitive Computation. Advance online publication. doi:10.1007/s12559-020-09787-5 PMID:33425044

Jaiswal, A. K., Tiwari, P., Kumar, S., Gupta, D., Khanna, A., \& Rodrigues, J. J. (2019). Identifying pneumonia in chest X-rays: A deep learning approach. Measurement, 145, 511-518. doi:10.1016/j.measurement.2019.05.076

Kaggle. (n.d.). COVID. https://www.kaggle.com/luisblanche/COVID

Khan, A. I., Shah, J. L., \& Bhat, M. M. (2020). Coronet: A deep neural network for detection and diagnosis of COVID-19 from chest x-ray images. Computer Methods and Programs in Biomedicine, 196, 105581. doi:10.1016/j.cmpb.2020.105581 PMID:32534344

Khatri, A., Jain, R., Vashista, H., Mittal, N., Ranjan, P., \& Janardhanan, R. (2020). Pneumonia Identification in Chest X-Ray Images Using EMD. In Trends in Communication, Cloud, and Big Data (pp. 87-98). Springer. doi:10.1007/978-981-15-1624-5_9

Kolhar, M., Al-Turjman, F., Alameen, A., \& Abualhaj, M. M. (2020). A Three Layered Decentralized IoT Biometric Architecture for City Lockdown during COVID-19 Outbreak. IEEE Access: Practical Innovations, Open Solutions, 8(1), 163608-163617. doi:10.1109/ACCESS.2020.3021983

Krause, J., Sapp, B., Howard, A., Zhou, H., Toshev, A., Duerig, T., Philbin, J., \& Fei-Fei, L. (2015). The unreasonable effectiveness of noisy data for fine-grained recognition. arXiv preprint arXiv:1511.06789. 
Krizhevsky, A., Sutskever, I., \& Hinton, G. E. (2012). Imagenet classification with deep convolutional neural networks. Advances in Neural Information Processing Systems, 1097-1105.

Lancet, . (2020). COVID-19: Too little, too late? Lancet, 395(10226), 755. doi:10.1016/S0140-6736(20)30522-5 PMID:32145772

Li, L., Qin, L., Xu, Z., Yin, Y., Wang, X., Kong, B., \& Cao, K. et al. (2020). Artificial intelligence distinguishes COVID-19 from community acquired pneumonia on chest CT. Radiology. Advance online publication. doi:10.1148/radiol.2020200905

Mahmud, M., Kaiser, M. S., \& Hussain, A. (2020). Deep learning in mining biological data. arXiv preprint arXiv:2003.00108.

Mahmud, M., Kaiser, M. S., Hussain, A., \& Vassanelli, S. (2018). Applications of deep learning and reinforcement learning to biological data. IEEE Transactions on Neural Networks and Learning Systems, 29(6), 2063-2079. doi:10.1109/TNNLS.2018.2790388 PMID:29771663

Mangal, A., Kalia, S., Rajgopal, H., Rangarajan, K., Namboodiri, V., Banerjee, S., \& Arora, C. (2020). CovidAID: COVID-19 Detection Using Chest X-Ray. arXiv preprint arXiv:2004.09803.

Muhammad, L. J., Algehyne, E. A., Usman, S. S., Ahmad, A., Chakraborty, C., \& Mohammed, I. A. (2021). Supervised Machine Learning Models for Prediction of COVID-19 Infection using Epidemiology Dataset. SN. Computer Science, 2(1), 11. doi:10.1007/s42979-020-00394-7 PMID:33263111

Peng, X., Xu, X., Li, Y., Cheng, L., Zhou, X., \& Ren, B. (2020). Transmission routes of 2019-nCoV and controls in dental practice. International Journal of Oral Science, 12(1), 1-6. doi:10.1038/s41368-020-0075-9 PMID:32127517

Rasheed, J. (2020). A Machine Learning-based framework for diagnosis of COVID-19 from Chest X-ray Images. Interdisciplinary Sciences, Computational Life Sciences. Advance online publication. doi:10.1007/s12539-02000403-6 PMID:33387306

Razai, M. S., Doerholt, K., Ladhani, S., \& Oakeshott, P. (2020). Coronavirus disease 2019 (covid-19): A guide for UK GPs. BMJ (Clinical Research Ed.), 368. doi:10.1136/bmj.m800 PMID:32144127

Sethy, P. K., \& Behera, S. K. (2020). Detection of coronavirus disease (covid-19) based on deep features. Preprints, 2020030300, 2020.

Sharon, J. J., Anbarasi, L. J., \& Raj, B. E. (2018, November). DPSO-FCM based segmentation and Classification of DCM and HCM Heart Diseases. In 2018 Fifth HCT Information Technology Trends (ITT) (pp. 41-46). IEEE. doi:10.1109/CTIT.2018.8649511

Song, Y., Zheng, S., Li, L., Zhang, X., Zhang, X., Huang, Z., . . Chong, Y. (2020). Deep learning enables accurate diagnosis of novel coronavirus (COVID-19) with CT images. medRxiv. 10.1101/2020.02.23.20026930

Srivastava, V. (2020). A Systematic Approach for the COVID-19 Prediction and Parameters Estimation. Personal and Ubiquitous Computing Journal. doi:10.1007/s00779-020-01462-8

Szegedy, C., Ioffe, S., Vanhoucke, V., \& Alemi, A. (2016). Inception-v4, inception-resnet and the impact of residual connections on learning. arXiv preprint arXiv:1602.07261.

Texas. (n.d.). Coronavirus. https://dshs.texas.gov/uploadedImages/Content/Consumer_and_External_Affairs/ coronavirus/banner.png

Varela-Santos, S., \& Melin, P. (2020). Classification of X-Ray Images for Pneumonia Detection Using Texture Features and Neural Networks. In Intuitionistic and Type-2 Fuzzy Logic Enhancements in Neural and Optimization Algorithms: Theory and Applications (pp. 237-253). Springer. doi:10.1007/978-3-030-35445-9_20

Worldometers. (n.d.). Coronavirus. https://www.worldometers.info/coronavirus/worldwide-graphs/

Xie, X., Zhong, Z., Zhao, W., Zheng, C., Wang, F., \& Liu, J. (2020). Chest CT for typical 2019-nCoV pneumonia: Relationship to negative RT-PCR testing. Radiology, 200343. doi:10.1148/radiol.2020200343

Zheng, C., Deng, X., Fu, Q., Zhou, Q., Feng, J., Ma, H., . . Wang, X. (2020). Deep learning-based detection for COVID-19 from chest CT using weak label. medRxiv. 10.1101/2020.03.12.20027185 